\title{
Classical Coding and the Cauchy-Schwarz Inequality
}

\author{
*Bas Janssens \\ October 2006 \\ * Mathematical Institute, Utrecht University
}

\begin{abstract}
In classical coding, a single quantum state is encoded into classical information. Decoding this classical information in order to regain the original quantum state is known to be impossible. However, one can attempt to construct a state which comes as close as possible. We give bounds on the smallest possible trace distance between the original and the decoded state which can be reached. We give two approaches to the problem: one starting from Keyl and Werner's no-cloning theorem [KW], and one starting from an operator-valued Cauchy-Schwarz inequality.
\end{abstract}

\section{Introduction}

There exist results which are possible in classical physics, yet forbidden in quantum mechanics. For instance, it is generally impossible to perform a joint measurement on two observables, or to clone one quantum state to two identical ones. This yields a class of problems: exactly how close can one approximate these desired, yet impossible results?

We examine this problem in the specific case of 'classical coding'. Impossibility of classical coding is the following statement: if a single quantum state is encoded into classical information, then it is impossible, starting from this classical information, to reconstruct the original quantum state.

In order to quantify how far one is removed from the ideal situation, we define $\Delta$ to be the worst case trace distance between the original and the decoded state. The impossibility of classical coding is then equivalent to $\Delta \neq 0$. The aim is now to investigate which values of $\Delta$ can be reached. We will do this in two different ways.

- The heart of this paper is the operator-valued Cauchy-Schwarz inequality, described in section 4 We will use it to obtain the bound $\Delta \geq(3-\sqrt{5}) / 4$.

- We will also attack the same problem using a no-cloning theorem [KW], which will yield the superior bound $\Delta \geq 1 / 3$.

The Cauchy-Schwarz method was used earlier in [J1 for the three quantum impossibilities called 'no measurement without disturbance', 'no measurement without decoherence' and 'no joint measurement'. In contrast with the case considered here, the bounds in these three cases did turn out to be sharp. The purpose of this paper is mainly to illustrate the flexibility of the Cauchy-Schwarz method. 
The article is structured as follows: in section 2 a short introduction to quantum probability theory is given. In section 3 the problem of classical coding is formulated in a rigorous mathematical fashion. In section 4 the Cauchy-Schwarz inequality is given, and applied to the problem of classical coding. This yields the bound $\Delta \geq(3-\sqrt{5}) / 4$. Section 5 provides the superior bound $\Delta \geq 1 / 3$, based on the no-cloning theorem. A short discussion of the results then follows in section 6

\section{Quantum Mechanics}

A quantummechanical system is described by a von Neumann algebra $\mathcal{A}$ of bounded operators on a Hilbert space $\mathcal{H}$, usually the algebra $B(\mathcal{H})$ of all bounded operators. Its state space is formed by the normalized density matrices $\mathcal{S}(\mathcal{A})=\{\rho \in \mathcal{A} ; \rho \geq 0, \operatorname{tr}(\rho)=1\}$. With the system in state $\rho \in \mathcal{S}(\mathcal{A})$, observation of a (Hermitean) observable $A \in \mathcal{A}$ is postulated to yield the average value $\operatorname{tr}(\rho A)$.

\subsection{Completely Positive maps}

The natural notion of a map between von Neumann-algebras is that of a completely positive (or CP for short) map.

Definition. Let $\mathcal{A}$ and $\mathcal{B}$ be von Neumann algebras. A weakly continuous map $T: \mathcal{B} \rightarrow \mathcal{A}$ is called Completely Positive (or CP for short) if it is linear, normalized (i.e. $T(I)=I$ ), positive (i.e. $T\left(X^{\dagger} X\right) \geq 0$ for all $\left.X \in \mathcal{B}\right)$ and if moreover the extension $I d_{n} \otimes T: M_{n} \otimes \mathcal{B} \rightarrow M_{n} \otimes \mathcal{A}$ is positive for all $n \in \mathbb{N}$, where $M_{n}$ is the algebra of complex $n \times n$-matrices.

Its dual $T^{*}: \mathcal{S}(\mathcal{A}) \rightarrow \mathcal{S}(\mathcal{B})$, defined by the requirement $\operatorname{tr}\left(T^{*}(\rho) X\right)=\operatorname{tr}(\rho T(X)) \forall X \in \mathcal{B}$, has a direct physical interpretation as an operation between quantum systems. $T$ is positive, linear and normalized. This is equivalent to $T^{*}$ being an affine map $\mathcal{S}(\mathcal{A}) \rightarrow \mathcal{S}(\mathcal{B})$. That is, each state $\rho \in \mathcal{S}(\mathcal{A})$ is again mapped to a state $T^{*}(\rho) \in \mathcal{S}(\mathcal{B})$, and for all $p \in[0,1]$ and $\rho_{1}, \rho_{2} \in \mathcal{S}(\mathcal{A})$, we have $p T^{*}\left(\rho_{1}\right)+(1-p) T^{*}\left(\rho_{2}\right)=T^{*}\left(p \rho_{1}+(1-p) \rho_{2}\right)$. This expresses the stochastic equivalence principle: a system which is in state $\rho_{1}$ with probability $p$ and in state $\rho_{2}$ with probability $(1-p)$ cannot be distinguished from a system in state $p \rho_{1}+(1-p) \rho_{2}$.

It is possible to extend the systems $\mathcal{A}$ and $\mathcal{B}$ under consideration with another system $M_{n}$, on which the operation acts trivially. Due to complete positivity, states in $\mathcal{S}\left(M_{n} \otimes \mathcal{A}\right)$ are once again mapped to states in $\mathcal{S}\left(M_{n} \otimes \mathcal{B}\right)$. This is of course a crucial property if one seeks to interpret $T^{*}$ as a physical map: the mere act of coupling the system $\mathcal{A}$ to another system $M_{n}$ may never cause negative probabilities. Surprisingly enough, there exist linear maps which are positive, but not completely positive. It is generally believed that any operation which can be physically implemented on a quantum system is described by a CP-map.

\subsection{Probability Spaces}

We are interested in quantum operations which take as input a quantum state, and yield a classical probability distribution as output.

In order to encompass this in our framework of CP-Maps and von Neumann algebras, we identify a classical probability space with a commutative von Neumann algebra. (See [M1].) A classical probability space $(\Omega, \Sigma, \mathbb{P})$ gives rise to $\mathcal{A}:=L^{\infty}(\Omega, \Sigma, \mathbb{P})$, the set of bounded measurable functions on $\Omega$ up to the equivalence $\sim$, with $f \sim g$ if $f=g$ almost surely. We think of these as the random variables. Since $f \in \mathcal{A}$ acts on the Hilbert space $L^{2}(\Omega)$ by multiplication, we can regard $\mathcal{A}$ as a commutative subalgebra of $B\left(L^{2}(\Omega)\right)$, which turns out 
to be weakly closed. The probability measure $\mathbb{P}$ of course induces the expectation $\mathbb{E}(f)=$ $\int_{\Omega} f(\omega) \mathbb{P}(d \omega)$ of a random variable. This yields a state $\rho_{\mathbb{P}}$ by requiring $\operatorname{tr}\left(\rho_{\mathbb{P}} M_{f}\right):=\mathbb{E}(f)$.

In short, a classical probability space corresponds to a commutative von Neumann algebra, and a probability measure corresponds to a state on that algebra. An operation $C^{*}$ which maps quantum states in $\mathcal{S}(B(\mathcal{H}))$ to classical probability distributions in $\mathcal{S}(\mathcal{A})$ must therefore be the dual of a CP-map $C: \mathcal{A} \rightarrow B(\mathcal{H})$.

For example, the direct measurement of an observable (= bounded Hermitean operator) $X$. With each Hermitean operator, there is associated a projection valued measure $P(d x)$, such that $X=\int_{\operatorname{Spec}(X)} x P(d x)$. The measurement of $X$ is then represented by the CPmap $L^{\infty}(\operatorname{Spec}(X)) \rightarrow B(\mathcal{H})$ defined by $f \mapsto \int_{\mathbf{S p e c}(X)} f(x) P(d x)$. In the dual (Schrödinger) picture, we then see that a state $\rho \in \mathcal{S}(B(\mathcal{H}))$ induces the probability distribution $\mathbb{P}(d x)=$ $\operatorname{tr}(\rho P(d x))$ on the spectrum of $X$. More generally, each CP-map $L^{\infty}(\Omega) \rightarrow B(\mathcal{H})$ corresponds to a Positive Operator Valued Measure, but we will not need this here.

Similarly, an operation $D^{*}$ which maps classical probability distributions into quantum states must be the dual of a CP-map $D: B(\mathcal{H}) \rightarrow \mathcal{A}$.

\section{Classical Coding}

By classical coding, we mean the following procedure. First, classical information is extracted from a quantum system. This is described by the coding map $C: \mathcal{A} \rightarrow B(\mathcal{H})$, with $\mathcal{A}=$ $L^{\infty}(\Omega)$ for some space $\Omega$. Then, on the basis of this classical information, the original state is reconstructed as well as possible by means of some decoding procedure. This is described by a CP-map $D: B(\mathcal{H}) \rightarrow \mathcal{A}$. In the dual picture, this gives

$$
\mathcal{S}(B(\mathcal{H})) \stackrel{C^{*}}{\rightarrow} \mathcal{S}(\mathcal{A}) \stackrel{D^{*}}{\rightarrow} \mathcal{S}(B(\mathcal{H})) .
$$

The coding procedure is flawless iff every state is reconstructed perfectly, i.e. iff $C \circ D$ : $B(\mathcal{H}) \rightarrow B(\mathcal{H})$ is the identity.

It is well known We M2 that perfect classical coding is impossible. So let us investigate how close we can come to perfection. Define $\Delta$ to be the maximum difference between input and output probability on a single event $P$, i.e.

$$
\Delta:=\sup _{\rho, P}\left|\operatorname{tr}\left(D^{*} C^{*}(\rho) P\right)-\operatorname{tr}(\rho P)\right|,
$$

where $\rho$ runs over $\mathcal{S}(B(\mathcal{H})$ ), and $P$ runs over the projections in $B(\mathcal{H})$. We give two reformulations of this definition (see[NC]). The first is $\Delta=\sup _{\rho}\left|D\left(\rho, D^{*} C^{*}(\rho)\right)\right|$, with $D(\rho, \tau)$ the trace distance or Kolmogorov distance $D(\rho, \tau)=\frac{1}{2} \operatorname{tr}(|\rho-\tau|)$ between $\rho$ and $\tau$. The second is $\Delta=\sup _{B}\|B-C D(B)\|$, where $B$ runs over the positive operators $0 \leq B \leq I$. The latter is most convenient, and we will use it in the remainder of the article.

We remark that $\Delta$ quantifies the quality of the coding procedure: a large value of $\Delta$ corresponds to a poor classical coding procedure, a small value of $\Delta$ corresponds to a good one. Furthermore, $\Delta=0$ if and only if all states are encoded perfectly. We now investigate how close to zero $\Delta$ can come.

\section{The Cauchy-Schwarz Method}

We start with a lemma which, in all its simplicity, is the cornerstone of a veritable zoo of quantum inequalities. (See J1 J2]. The special case $(A, A)=0 \Rightarrow(A, B)=0$ is older, and due to Werner We.) 
Lemma 1 (Cauchy-Schwarz) Let $\mathcal{A}, \mathcal{B}$ be von Neumann algebras, and let $(\cdot, \cdot): \mathcal{A} \times \mathcal{A} \rightarrow$ $\mathcal{B}$ be a positive semidefinite sesquilinear form. That is, it is linear in the second argument, $(A, B)^{\dagger}=(B, A)$ for all $A, B \in \mathcal{A}$, and $(A, A) \geq 0$ for all $A \in \mathcal{A}$. Then $\|\Re(A, B)\|^{2} \leq$ $\|(A, A)\|\|(B, B)\|$ and $\|\Im(A, B)\|^{2} \leq\|(A, A)\|\|(B, B)\|$ for all $A, B \in \mathcal{A}$.

\section{Proof}

For $A, B \in \mathcal{A}$ and $\lambda \in \mathbb{C}$, we have (with $\Re X:=\left(X+X^{\dagger}\right) / 2$ the 'real' and $\Im X:=\left(X-X^{\dagger}\right) / 2 i$ the 'imaginary' part):

$$
0 \leq(A-\lambda B, A-\lambda B)=(A, A)-2 \Re \lambda(A, B)+|\lambda|^{2}(B, B) .
$$

If $(A, A)=0$ and $(B, B)=0$, the lemma follows immediately from (1). If not, assume that $(B, B) \neq 0$, exchanging the roles of $A$ and $B$ if necessary. Choose $\lambda= \pm\|\Re(A, B)\| /\|(B, B)\|$, so that (1) becomes the operator inequality

$$
\pm 2 \Re(A, B)\|\Re(A, B)\| /\|(B, B)\| \leq(A, A)+(B, B)\|\Re(A, B)\|^{2} /\|(B, B)\|^{2} .
$$

In particular, the spectrum of the operator on the l.h.s. is contained in $[-\|R\|,\|R\|]$, with $R$ the r.h.s. operator. Thus $2\|\Re(A, B)\|^{2} /\|(B, B)\| \leq\|R\|$, and $\|R\| \leq\|(A, A)\|+\|\Re(A, B)\|^{2} /\|(B, B)\|$. This yields $\|\Re(A, B)\|^{2} \leq\|(A, A)\|\|(B, B)\|$, as required. Since $\Re(i A, B)=\Im(A, B)$ holds, we also have $\|\Im(A, B)\|^{2} \leq\|(A, A)\|\|(B, B)\|$.

For example, each CP-map $T: \mathcal{A} \rightarrow \mathcal{B}$ induces a positive semidefinite sesquilinear form by $(A, B)_{T}:=T\left(A^{\dagger} B\right)-T(A)^{\dagger} T(B)$. Indeed, according to Stinespring's theorem (see St]), we can assume without loss of generality that $T$ is of the form $T(A)=V^{\dagger} A V$, with $\|V\| \leq 1$. Then $(A, A)_{T}=V^{\dagger} A^{\dagger}\left(I-V V^{\dagger}\right) A V=\left(\sqrt{\left(I-V V^{\dagger}\right)} A V\right)^{\dagger} \sqrt{\left(I-V V^{\dagger}\right)} A V \geq 0$. The sesquilinearity is clear.

We use the Cauchy-Schwarz inequality to obtain a the bound on $\Delta$ for any classical coding procedure. (The proof is inspired by the 'impossibility of classical coding' version in [M2.)

Theorem 2 Let $\mathcal{A}$ be a commutative von Neumann-algebra, and let $B(\mathcal{H})$ be the algebra of bounded operators on a Hilbert space $\mathcal{H}$ of dimension $>1$. Let $C: \mathcal{A} \rightarrow B(\mathcal{H})$ and $D: \mathcal{B}(\mathcal{H}) \rightarrow$ $\mathcal{A}$ be CP-maps. Let $\Delta:=\sup \{\|B-C \circ D(B)\|: 0 \leq B \leq I\}$. Then $\Delta \geq(3-\sqrt{5}) / 4$.

Proof

Take two orthogonal vectors $\psi, \phi \in \mathcal{H}$, and define $X$ to be the projection on $\psi$, and $Y$ the projection on $(\psi+\phi) / \sqrt{2}$. We have $\|[X, Y]\|=1 / 2$. Since $\mathcal{A}$ is Abelian, we have $D(X) D(Y)=$ $D(Y) D(X)$, and we can write

$$
\begin{aligned}
{[X, Y]=} & {[X, Y]-C D([X, Y])+} \\
& C(D(X Y)-D(X) D(Y))- \\
& C(D(Y X)-D(Y) D(X)) .
\end{aligned}
$$

We will bound the r.h.s. in terms of $\Delta$. Remembering that the l.h.s. is at least $1 / 2$ in norm will then yield a minimum value on $\Delta$.

We start with (2). Like any antihermitean operator, $[X, Y]$ can be written as $[X, Y]=$ $i\left(A_{+}-A_{-}\right)$, with $0 \leq A_{ \pm} \leq\|[X, Y]\| I=\frac{1}{2} I$. Therefore, we have $\|[X, Y]-C D([X, Y])\| \leq$ $\Delta\left\|A_{+}\right\|+\Delta\left\|A_{-}\right\| \leq \Delta$.

We then proceed with (3). Consider the positive semidefinite sesquilinear form $(X, Y):=$ $C\left(D\left(X^{\dagger} Y\right)-D(X)^{\dagger} D(Y)\right)$, in terms of which the expression (3) equals $2 i \Im(X, Y)$. It is positive as the concatenation of $(\cdot, \cdot)_{D}: B(\mathcal{H}) \times \mathcal{B}(\mathcal{H}) \rightarrow \mathcal{A}$ and the positive map $C: \mathcal{A} \rightarrow$ $B(\mathcal{H})$.

According to Lemma 1 we have $\|2 i \Im(X, Y)\| \leq 2 \sqrt{\|(X, X)\|\|(Y, Y)\|}$. Now $(X, X)=$ $(X, X)_{C D}-(D(X), D(X))_{C} \leq(X, X)_{C D}$, and a similar expression holds for $Y$. 
Since $X^{\dagger} X=X$, we have $(X, X)_{C D}=C D(X)(I-C D(X))$. Since $\|X-C D(X)\| \leq \Delta$, and $X$ has spectrum $\{0,1\}$, the spectrum of $C D(X)$ is contained in $[0, \Delta] \cup[1-\Delta, 1]$. (Recall that $0 \leq C D(X) \leq I$.) The spectrum of $C D(X)(I-C D(X))$ therefore lies within $[0, \Delta(1-\Delta)]$, so that $\left\|(X, X)_{C D}\right\| \leq \Delta(1-\Delta)$. The same holds for $Y$ of course. Putting this together, we bound (3) as $\|2 i \Im(X, Y)\| \leq 2 \Delta(1-\Delta)$.

We conclude that $1 / 2=\|[X, Y]\| \leq \Delta+2 \Delta(1-\Delta)$, or $(\Delta-3 / 4)^{2} \leq 5 / 16$. Thus $\Delta \geq$ $(3-\sqrt{5}) / 4$, which was to be proven.

\section{The No-Cloning method}

It is easy to obtain a better bound from the no-cloning theorem of Keyl and Werner [KW]. The idea is, that each classical coding procedure yields a cloning machine. All one has to do is to 'decode' the classical information an arbitrary amount $M$ of times, rather than just once.

To be more explicit, suppose that we are in the finite setting: $\mathcal{H}=\mathbb{C}^{d}$ is a finite-dimensional Hilbert space, and $\mathcal{A}=L^{\infty}(\Omega)$ with $\Omega=\{1,2, \ldots, n\}$. The 'diagonal' map $\Omega \rightarrow \Omega^{M}: i \mapsto$ $(i, \ldots, i)$ induces the 'classical cloning' map $K: \mathcal{A}^{\otimes M} \rightarrow \mathcal{A}$, i.e. $(K f)(i):=f(i, \ldots, i)$. (Note that for e.g. $\Omega=\mathbb{R}$, a cloning map poses difficulties.)

The composition $T:=C \circ K \circ D^{\otimes M}$, mapping $B\left(\mathbb{C}^{d}\right)^{\otimes M}$ to $B\left(\mathbb{C}^{d}\right)$, is a so-called $1 \rightarrow M$ cloner. By construction of $T$, it is clear that $T(I \otimes \ldots \otimes B \otimes \ldots \otimes I)=C D(B)$. The main result of $[\mathrm{KW}]$ then says that $\sup _{0 \leq B \leq I}\|T(I \otimes \ldots \otimes B \otimes \ldots \otimes I)-B\| \geq \frac{(M-1)}{M} \frac{d-1}{d+1}$. Since $M \in \mathbb{N}^{+}$was arbitrary, this implies $\Delta \geq \frac{d-1}{d+1}$. The best possible coding occurs for $d=2$, when $\Delta \geq \frac{1}{3}$.

\section{Discussion}

The number $(3-\sqrt{5}) / 4 \approx 0.19$, obtained from the Cauchy-Schwarz inequality, is inferior to the $\frac{1}{3} \approx 0.33$ from the no-cloning theorem, in usefulness as well as in the standard order on $\mathbb{R}$.

Although it yields inferior results for classical coding, the Cauchy-Schwarz method is simpler. At least taking into account the fairly heavy machinery needed in $\mathrm{KW}$. It is also very flexible. For any kind of quantum impossibility, ranging from 'no classical coding' to 'no measurement without disturbance', and from 'decoherence after measurement' to 'no joint measurement', the Cauchy-Schwarz inequality yields a bound which quantifies how far one is removed from the ideal, impossible situation. (See [J1] J2].)

Surprisingly enough, in the cases of 'no measurement without decoherence' 'no measurement without disturbance' and 'no joint measurement', this bound becomes sharp in the sense that there exist quantum operations which reach it. Furthermore, as far as I am aware, these three bounds cannot be obtained in any other way.

In conclusion, the Cauchy-Schwarz method yields quantitative bound for a variety of quantum impossibilities, including classical coding. Although this is not the case for classical coding, many of these bounds turn out to be sharp.

\section{References}

[J1] B. Janssens, 'Unifying Decoherence and the Heisenberg Principle', www.arxiv.org/quant-ph/0606093.

[J2] B. Janssens, 'Quantum Measurement, a Coherent Description', master's thesis, www . arxiv. org/quant-ph/0503009. 
[KW] M. Keyl, R. Werner, 'Optimal Cloning of Pure States, Judging Single Clones', J.Math.Phys. 40 3283-3299, (1999).

[NC] M. Nielsen, I. Chuang, 'Quantum Computation and Quantum Information', Cambridge University Press, (2000).

[M1] J.D.M. Maassen, 'Quantum Probability Applied to the Damped Harmonic Oscillator', www . arxiv.org/quant-ph/0411024, (2004).

[M2] J.D.M. Maassen, 'Quantum Probability, Quantum Information and Quantum Computing', www.math.kun.nl/medewerkers/maassen, (2004).

[St] W.F. Stinespring, 'Positive Functions on $C^{*}$-algebras', Proc. Amer. Math. Soc. 6, 211216, (1955).

[We] R. Werner, 'Quantum Information Theory - an Invitation', Springer Tracts in Modern Physics, 173, 14-57, (2001). 